\title{
Do polymorphisms in protein kinase catalytic subunit alpha-1 gene associated with cancer susceptibility? a meta-analysis and systematic review
}

\author{
Jialin Meng ${ }^{1,2,3}$, Xinyao Fan ${ }^{4}$, Meng Zhang ${ }^{1,2,3}$, Zongyao Hao ${ }^{1,2,3}$ and Chaozhao Liang ${ }^{1,2,3^{*}}$ (1)
}

\begin{abstract}
Background: Currently, several studies have demonstrated that PRKAA1 polymorphisms conduce to the development of cancer. PRKAA1 gene encodes the AMP-activated protein kinase summit-a1, and plays an important role in cell metabolism. Thus, we performed a systematic review and meta-analysis of all enrolled eligible case-control studies to obtain a precise correlation between PRKAA1 polymorphism and cancer susceptibility.

Methods: Extensive retrieve was performed in Web of Science, Google Scholar, PubMed, EMbase, CNKI and Wanfang databases up to August 26, 2018. Odds ratios (ORs) and 95\% Cls were performed to evaluate the overall strength of the associations in five models, as well as in subgroup analyses, stratified by ethnicity, cancer type or source of control. Q-test, Egger's test and Begg's funnel plot were applied to evaluate the heterogeneity and publication bias. In-silico analysis was performed to demonstrate the relationship of PRKAA1 expression correlated with cancer tissues and survival time.

Results: Twenty-two case-control studies from 14 publications were enrolled, with 17,068 cases and 20,871 controls for rs13361707, and 2514 cases and 3193 controls for rs10074991. Overall, we identified that the PRKAA1 rs13361707 polymorphism is not significantly associated with cancer susceptibility under all five genetic models. For rs10074991, we revealed a significant decrease risk in allelic comparison model ( $B$ vs. A: $O R=0.774,95 \% \mathrm{Cl}=0.642-0.931$, $P_{\text {Adjust }}=3.376^{*} 10^{-2}$ ), heterozygote comparison model (BA vs. $\mathrm{AA}: \mathrm{OR}=0.77995 \% \mathrm{Cl}=0.691-0.877, P_{\text {Adjust }}=9.86^{*} 10^{-10}{ }$ ), and dominant genetic model (BB + BA vs. AA: $\mathrm{OR}=0.69795 \% \mathrm{Cl}=0.533-0.912, P_{\text {Adjust }}=4.211^{*} 10^{-2}$;). Evidence from TCGA database and GTEx projects indicated that the expression of PRKAA1 in gastric cancer tissue is higher, compared to normal stomach tissue, as well as it in breast cancer and esophageal squamous cell carcinoma. However, the Kaplan-Meier estimate showed that there is no significant difference of OS and RFS between the low and high PRKAA1 TPM groups in gastric cancer, breast cancer, and esophageal carcinoma.
\end{abstract}

Conclusions: To sum up, PRKAA1 rs13361707 polymorphism is not participant with the increased risk of cancer, while the A allele of PRKAA1 rs10074991 revealed a significant decrease risk.

Keywords: PRKAA1, Polymorphism, Cancer, Meta-analysis

\footnotetext{
* Correspondence: liang_chaozhao@ahmu.edu.cn

${ }^{1}$ Department of Urology, The First Affiliated Hospital of Anhui Medical

University, No. 218th, Jixi Road, Hefei 230022, Anhui, China

${ }^{2}$ Institute of Urology, Anhui Medical University, No. 218th, Jixi Road, Hefei

230022, Anhui, China

Full list of author information is available at the end of the article
}

(c) The Author(s). 2018 Open Access This article is distributed under the terms of the Creative Commons Attribution 4.0 International License (http://creativecommons.org/licenses/by/4.0/) which permits unrestricted use, distribution, and reproduction in any medium, provided you give appropriate credit to the original author(s) and the source, provide a link to the Creative Commons license, and indicate if changes were made. The Creative Commons Public Domain Dedication waiver (http://creativecommons.org/publicdomain/zero/1.0/) applies to the data made available in this article, unless otherwise stated. 


\section{Background}

It is well known that hereditary materials and environmental aspects could influence the risks and take a critical part in the tumorigenesis of numerous cancers $[1,2]$, nevertheless, the risk attributable to each cancer is indistinct. Of them, an important one is AMP-activated protein kinase (AMPK). As a heterotrimeric protein complex, AMPK always made up of one $\alpha, \beta$ and $\gamma$ subunit, respectively, and the $39 \mathrm{~kb}$-long encodes gene (PRKAA1) of $\alpha$-subunit is located at chromosome 5 p12.1 $[3,4]$. Numerous pivotal cell metabolic enzymes are regulated by AMPK, through its function of Ser/Thr phosphorylation [5].AMPK induce the important inhibition of tumorigenesis through multifaceted ways. Firstly, AMPK activation contributes to the inhibition in fatty acid biosynthesis and cholesterol biosynthesis, as well as to the promotion of fatty acid oxidation, therefore, against to the intracellular lipid accumulation and insulin resistance development in non-adipose tissues [6]. Secondly, another important tumorigenesis related signaling pathway, the mammalian target of rapamycin complex 1 (mTORC1), can be inhibited by the activation of AMPK [7]. Thirdly, AMPK activation can result in the G1 phase cell cycle arrest, and further impact the cell proliferation through the p53-p21 axis [8].

Most of the publications concerned about PRKAA1 polymorphisms focused on its significantly positive association with gastric cancer (GC) [9-11], as well as on breast cancer [12], but there are also some negative results [13-16]. These conflicting results might be partially affect by the cancer type, origin of control, or sample size. Herein, we managed the meta-analysis to assess whether PRKAA1 polymorphisms affect susceptibility of cancer.

\section{Methods}

\section{Identification and selection of eligible studies}

Comprehensive literature search on Web of Science, Google Scholar, PubMed, EMbase, CNKI and Wanfang databases was conducted to draw out all eligible case-control studies, and the latest search date is August 26, 2018. The following are valid keyword search strings: (PRKAA1) AND (polymorphism OR SNP OR variant OR mutation OR allele) AND (cancer OR tumor OR tumour OR carcinoma OR neoplasm OR malignancy). What's more, we also manually retrieved the references of reviews or original research on this issue to identify additional studies. For these republished and overlapping studies, we enrolled the most recently published articles or case-control studies with a maximum number of subjects.

\section{Enrolled criteria and excluded criteria}

All the eligible studies were enrolled following the details: 1) assess the correlation between the polymorphisms in
PRKAA1 and cancer susceptibility; 2) case-control studies; 3) demonstrate the frequency of genotypes of all cases and controls, or could obtain it via calculating. However, studies would be excluded if they meet the items: 1) animal studies, meta-analysis, comments, reviews or case reports; 2) no efficient data of the genotype frequency; 3) repetitive publications; 4) the research contents were concerned about other disorders instead of cancers.

\section{Extraction of data}

Extraction of data were independently completed by two authors (Jialin Meng, Xinyao Fan), and all disagreements were finally obtained a consensus after discussion. The following items from the eligible case-control studies were extracted: SNP code, first author, year of publication, ethnicity, cancer type, genotyping method, source of control and genotype frequency. For the control group of each study, we definition it as population-based or hospital-based from whether it is collected from physical examination or just no-cancer patients, and ethnicity was distinguished as "Caucasian" or "Asian".

\section{Statistical analysis}

We performed the meta-analyses in the pool, ORs with corresponding 95\% CI was recorded to evaluated the strength of the correlation between PRKAA1 polymorphisms (rs13361707, rs10074991) and cancer susceptibility, the $P$ value was adjusted by Bonferroni corrections, $P_{\text {Adjust }}=P_{Z} * 5$ models [17]. We pooled the ORs for allelic comparison model (B vs. A), heterozygote comparison model (BA vs. AA), homozygote comparison model (BB vs. $\mathrm{AA})$, dominant genetic model $(\mathrm{BB}+\mathrm{BA}$ vs. $\mathrm{AA})$ and recessive genetic model ( $\mathrm{BB}$ vs. $\mathrm{BA}+\mathrm{AA})$. We further applied Q-test to assess the between study heterogeneity [18]. When the $P$ value of the $\mathrm{Q}$-test was $>0.1$, we selected the Mantel-Haenszel method (fixed model) to evaluate the pooled OR estimate; On the contrast, the Der Simonian and Laird method (random-effect model) was preferred to evaluated the $P$ value $<0.1$ group $[19,20]$. In the subgroup of ethnicity and source of control, stratified analyses were also conducted. The Hardy-Weinberg equilibrium (HWE) for the control group was assessed by a professional web-based program (https://wpcalc.com/en/equilibrium-hardy-weinberg/), the $P$-value $>0.05$ suggested a HWE balance for the control group. In order to appraise the stability of the meta-analyses, we managed sensitivity analyses by excluding each case-control study and observe whether it influence the pooled ORs and 95\% CI [21]. What's more, Begg's funnel plot and Egger's test was also conducted to avoid any potential publication bias [22, 23]. All the analysis results were calculated by STATA 12.0 (version 12.0; Stata Corporation). and $P<0.05$ was regarded as statistically significant. 


\section{In-silico analysis}

To further explore whether the expression of PRKAA1 affect tumorigenesis, we tried to search some evidence from the public TCGA database and GTEx projects, and a newly developed interactive website, GEPIA (http:// gepia.cancer-pku.cn/), was applied to draw out it [24].

\section{Results}

\section{Characteristics of studies}

Figure 1 displays the process of searching, a total of 320 publications was firstly retrieved from PubMed, EMbase, Web of Science, CNKI and Wanfang databases. After reviewed the abstract, 54 publications were selected for a further evaluation, however, 30 of them were expurgated because of that they were duplicated studies, case-only studies, or less of efficient data. Finally, 22 case-control studies from 14 publications were enrolled in our study, 17,068 cases and 20,871 controls for rs13361707 [9-16, 25-30], while 2514 cases and 3193 controls for rs10074991 $[10,12,27]$ (Table 1, Additional file 1: Table S1). There are 20 case-control studies from Asian descendants, and 2 from Caucasian. Diagnose of tumor was confirmed histologically or pathologically. As to HWE, only three studies were not conformed to it, while the other 19 case-control studies conformed to it. In addition, the result of appraise the quality of study by Newcastle-Ottawa Scale (NOS) is filled in Additional file 1: Table S2 [31], and Additional file 1: Table S3 shows the result of PRISMA2009 checklist [32].

\section{Main results}

Table 2 shows the summarized details and results of the meta-analyses. We identified that the rs 13361707 polymorphism is not related to the susceptibility of cancer under all five genetic models in the overall population (B vs. $\mathrm{A}: \mathrm{OR}=0.900,95 \% \mathrm{CI}=0.776-1.042, P_{\text {Adjust }}=0.795$; $\mathrm{BB}$ vs. $\mathrm{AA}: \mathrm{OR}=0.810,95 \% \mathrm{CI}=0.601-1.092, P_{\text {Adjust }}=$ 0.830 ; $\mathrm{BA}$ vs. $\mathrm{AA}: \mathrm{OR}=0.900,95 \% \mathrm{CI}=0.768-1.054$, $P_{\text {Adjust }}=0.960 ; \mathrm{BA}+\mathrm{AA}$ vs. $\mathrm{AA}: \mathrm{OR}=0.569,95 \% \mathrm{CI}=$ $0.711-1.061, P_{\text {Adjust }}=0.840$; and $\mathrm{BB}$ vs. $\mathrm{BA}+\mathrm{AA}: \mathrm{OR}=$ $\left.0.965,95 \% \mathrm{CI}=0.714-1.071, P_{\text {Adjust }}=0.965\right)$, and the stratified analysis also indicated no relationships in the

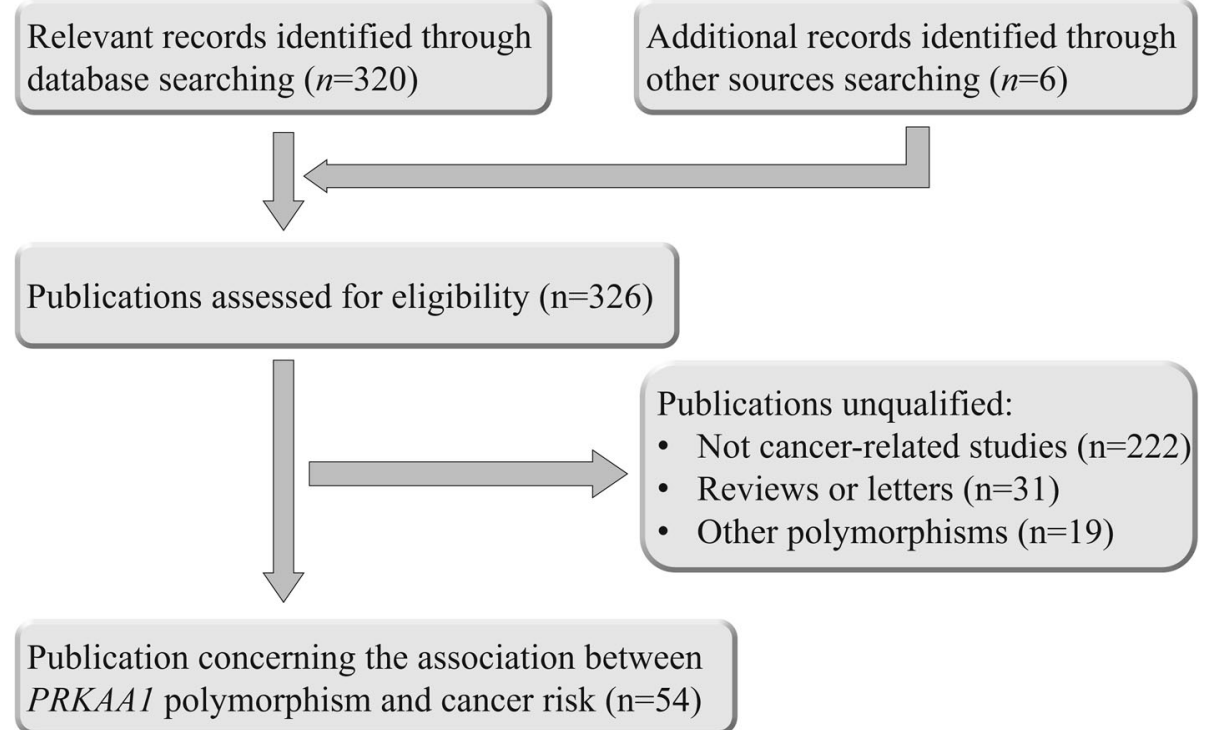

PRKAAl polymorphism and cancer risk $(\mathrm{n}=54)$

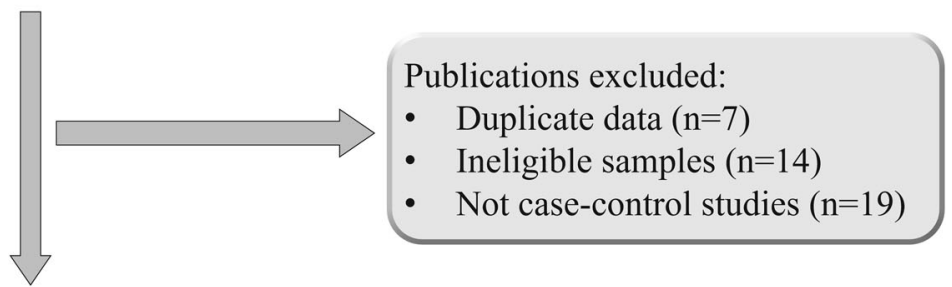

Publications included in the meta-analysis $(\mathrm{n}=14)$

- 3 publications including 3 case-control studies for PRKAA1 rs 10074991

- 13 publications including 19 case-control studies for PRKAA1 rs13361707

Fig. 1 Flow chart showing the study selection procedure 
Table 1 Characteristics of the enrolled studies on PRKAA1 polymorphisms and cancer

\begin{tabular}{|c|c|c|c|c|c|c|c|c|c|c|c|c|c|}
\hline \multirow[t]{2}{*}{ SNP } & \multirow[t]{2}{*}{ First author } & \multirow[t]{2}{*}{ Year } & \multirow[t]{2}{*}{ Ethnicity } & \multirow[t]{2}{*}{ Genotyping Method } & \multirow{2}{*}{$\begin{array}{l}\text { Source of } \\
\text { Control }\end{array}$} & \multirow{2}{*}{$\begin{array}{l}\text { Cancer } \\
\text { Type }\end{array}$} & \multicolumn{3}{|l|}{ Csae } & \multicolumn{3}{|c|}{ Control } & \multirow[t]{2}{*}{ HWE } \\
\hline & & & & & & & PAA & PAB & PBB & HAA & $\mathrm{HAB}$ & $\mathrm{HBB}$ & \\
\hline rs10074991 & Campa et al. & 2011 & Caucasian & HapMap & $\mathrm{HB}$ & $B C$ & 654 & 445 & 94 & 960 & 764 & 149 & Y \\
\hline rs10074991 & Kim et al. & 2014 & Asian & GoldenGate assay & PB & GC & 136 & 242 & 97 & 94 & 244 & 136 & Y \\
\hline rs10074991 & Eom et al. & 2016 & Asian & GoldenGate assay & PB & GC & 248 & 421 & 177 & 169 & 429 & 248 & Y \\
\hline rs13361707 & Shi et al. & 2011 & Asian & AGWHSA 6.0 chips & PB & GC & 160 & 517 & 302 & 607 & 1154 & 507 & Y \\
\hline rs13361707 & Shi et al. & 2011 & Asian & AGWHSA 6.0 chips & PB & GC & 371 & 941 & 561 & 578 & 1034 & 464 & Y \\
\hline rs13361707 & Shi et al. & 2011 & Asian & AGWHSA 6.0 chips & PB & GC & 237 & 675 & 480 & 392 & 745 & 376 & Y \\
\hline rs13361707 & Shi et al. & 2011 & Asian & AGWHSA 6.0 chips & PB & GC & 223 & 447 & 225 & 713 & 1616 & 898 & Y \\
\hline rs13361707 & Shi et al. & 2011 & Asian & AGWHSA 6.0 chips & PB & GC & 724 & 1221 & 459 & 713 & 1616 & 898 & Y \\
\hline rs13361707 & Li et al. & 2013 & Asian & TaqMan & PB & GC & 97 & 167 & 71 & 67 & 165 & 102 & Y \\
\hline rs13361707 & Song et al. & 2013 & Asian & HRM-PCR & PB & GC & 909 & 1654 & 682 & 377 & 846 & 477 & Y \\
\hline rs13361707 & Dai et al. & 2014 & Asian & TaqMan & PB & ESCC & 460 & 1054 & 558 & 507 & 1144 & 603 & Y \\
\hline rs13361707 & Wu et al. & 2014 & Asian & Multiplex SNaPshot SNP & PB & GC & 54 & 115 & 48 & 86 & 209 & 133 & Y \\
\hline rs13361707 & Kim et al. & 2014 & Asian & GoldenGate assay & PB & GC & 137 & 241 & 97 & 96 & 242 & 135 & Y \\
\hline rs13361707 & Sun et al. & 2014 & Caucasian & TapMan & $\mathrm{HB}$ & GC & 79 & 45 & 6 & 68 & 48 & 8 & Y \\
\hline rs13361707 & Dong et al. & 2015 & Asian & iMLDR & PB & GC & 37 & 68 & 62 & 54 & 91 & 41 & Y \\
\hline rs13361707 & Dong et al. & 2015 & Asian & iMLDR & PB & NSCLC & 41 & 71 & 46 & 54 & 91 & 41 & Y \\
\hline rs13361707 & Dong et al. & 2015 & Asian & iMLDR & PB & ESCC & 33 & 51 & 23 & 54 & 91 & 41 & Y \\
\hline rs13361707 & Qiu et al. & 2015 & Asian & TaqMan & PB & GC & 344 & 571 & 209 & 273 & 565 & 356 & Y \\
\hline rs13361707 & Zhang et al. & 2016 & Asian & MALDITOF & $\mathrm{HB}$ & GC & 23 & 27 & 10 & 10 & 34 & 16 & Y \\
\hline rs13361707 & Eom et al. & 2016 & Asian & GoldenGate assay & PB & GC & 249 & 421 & 176 & 174 & 424 & 248 & Y \\
\hline rs13361707 & Yuan et al. & 2016 & Asian & PCR & $\mathrm{HB}$ & GC & 31 & 59 & 26 & 28 & 49 & 25 & Y \\
\hline rs13361707 & Cai et al. & 2017 & Asian & KASP & PB & GC & 172 & 213 & 88 & 98 & 246 & 143 & $Y$ \\
\hline
\end{tabular}

GC Gastric cancer, BC Breast cancer, ESCC Esophageal squamous cell carcinoma, NSCLC Non-small cell lung cancer, HB Hospital based, PB Population based, HWE Hardy Weinberg Equilibrium

subgroups of cancer type, ethnicity and source of control (Fig. 2). For rs10074991, we revealed a significant decrease risk in allelic comparison model (B vs. A: OR = $0.774,95 \% \mathrm{CI}=0.642-0.931, P_{\text {Adjust }}=3.376 * 10^{-2}$ ), heterozygote comparison model (BA vs. AA: $\mathrm{OR}=0.779$ $95 \% \mathrm{CI}=0.691-0.877, P_{\text {Adjust }}=1.948 * 10^{-4} ;$ ), and dominant genetic model (BB + BA vs. AA: $\mathrm{OR}=0.69795 \% \mathrm{CI}=$ $0.533-0.912, P_{\text {Adjust }}=4.211^{*} 10^{-2}$;) (Fig. 3), and the subgroup of Asian people in rs10074991 shown a prevent potential for tumorigenesis in all five genetic models (B vs. $\mathrm{A}: \mathrm{OR}=0.704, \quad 95 \% \mathrm{CI}=0.632-0.785, \quad P_{\text {Adjust }}=$ $1.085 * 10-9 ; \quad$ BB vs. $\mathrm{AA}: \mathrm{OR}=0.489,95 \% \mathrm{CI}=0.489$ $0.392-0.609, \quad P_{\text {Adjust }}=9.86 * 10-10 ;$ BA vs. AA: $\mathrm{OR}=$ $0.675,95 \% \mathrm{CI}=0.558-0.816, P_{\text {Adjust }}=2.473^{*} 10-4 ; \quad \mathrm{BA}$ + AA vs. $\mathrm{AA}: \mathrm{OR}=0.607,95 \% \mathrm{CI}=0.507-0.727, P_{\text {Adjust }}=$ $2.691^{*} 10-7$; and $\mathrm{BB}$ vs. $\mathrm{BA}+\mathrm{AA}: \mathrm{OR}=0.638,95 \% \mathrm{CI}=$ $\left.0.534-0.762, P_{\text {Adjust }}=3.876 * 10-6\right)$.

\section{Sensitivity and publication bias analysis}

Sensitivity analysis by delete each signal study were performed to assess whether it influence the overall ORs results. The sensitivity analysis results conducted by all five genetic models were displayed in Fig. 4 and Additional file 1: Table S4, no signal study significantly influence the overall ORs results. On the besides, potential publication bias of enrolled case-control studies was appraised by Begg's funnel plot and Egger's test, no publication bias was revealed in both rs13361707 and rs10074991 (Additional file 1: Figures S1 and S2, Table S5).

To further explore whether the expression of PRKAA1 affect tumorigenesis, we tried to search some evidence from the public TCGA database and GTEx projects, and a newly developed interactive website, GEPIA (http:// gepia.cancer-pku.cn/), was applied to draw out it.

\section{In-silico analysis}

Evidence from TCGA database and GTEx projects indicated that the expression level of PRKAA1 in gastric cancer tissue is higher, when compared to normal stomach tissue (TPM $=54.3$ vs. $29.2, P<0.01$,), as well as in breast cancer (TPM $=23.1$ vs. $29.8, P<0.01)$ and esophageal squamous cell carcinoma (TPM $=49.6$ vs. $14.9, P<$ 0.01) (Fig. 5a). In addition, we analyzed whether the 
Table 2 Results of meta-analysis for polymorphisms in and cancer susceptibility

\begin{tabular}{|c|c|c|c|c|c|c|c|}
\hline Comparison & Subgroup & N & $P_{H}$ & $P_{z}$ & $P_{\text {Adjust }}$ & Random (OR, 95\%Cl) & Fixed $(\mathrm{OR}, 95 \% \mathrm{Cl})$ \\
\hline \multicolumn{8}{|l|}{ rs10074991 } \\
\hline B VS. A & overall & 3 & 0.006 & $6.752^{*} 10^{-3}$ & $3.376^{*} 10^{-2^{*}}$ & $0.774(0.642-0.931)$ & $0.795(0.735-0.861)$ \\
\hline B VS. A & Asian & 2 & 0.908 & $2.169^{*} 10^{-10}$ & $1.085^{*} 10^{-9^{*}}$ & $0.704(0.632-0.785)$ & $0.704(0.632-0.785)$ \\
\hline$B B$ vs. AA & overall & 3 & 0.002 & $2.787^{*} 10^{-2}$ & 0.139 & $0.610(0.393-0.946)$ & $0.627(0.528-0.745)$ \\
\hline BB vs. AA & Asian & 2 & 0.954 & $1.972^{*} 10^{-10}$ & $9.86^{*} 10^{-10^{*}}$ & $0.489(0.392-0.609)$ & $0.489(0.392-0.609)$ \\
\hline BA vs. AA & overall & 3 & 0.163 & $3.895 * 10^{-5}$ & $1.948^{*} 10^{-4^{*}}$ & $0.755(0.633-0.900)$ & $0.779(0.691-0.877)$ \\
\hline BA vs. AA & Asian & 2 & 0.903 & $4.946^{*} 10^{-5}$ & $2.473^{*} 10^{-4^{*}}$ & $0.675(0.558-0.816)$ & $0.675(0.558-0.816)$ \\
\hline $\mathrm{BB}+\mathrm{BA}$ vs. $\mathrm{AA}$ & overall & 3 & 0.011 & $8.421 * 10^{-3}$ & $4.211^{*} 10^{-2^{*}}$ & $0.697(0.533-0.912)$ & $0.752(0.672-0.842)$ \\
\hline$B B+B A$ vs. $A A$ & Asian & 2 & 0.900 & $5.381 * 10^{-8}$ & $2.691^{*} 10^{-7^{*}}$ & $0.607(0.507-0.727)$ & $0.607(0.507-0.727)$ \\
\hline$B B$ vs. $B A+A A$ & overall & 3 & 0.029 & $3.610^{*} 10^{-2}$ & 0.181 & $0.737(0.554-0.980)$ & $0.729(0.628-0.846)$ \\
\hline$B B$ vs. BA+AA & Asian & 2 & 0.999 & $7.752^{*} 10^{-7}$ & $3.876^{*} 10^{-6^{*}}$ & $0.638(0.534-0.762)$ & $0.638(0.534-0.762)$ \\
\hline \multicolumn{8}{|l|}{ rs13361707 } \\
\hline B VS. A & overall & 19 & $6.416^{*} 10^{-72}$ & 0.159 & 0.795 & $0.900(0.776-1.042)$ & $0.931(0.904-0.959)$ \\
\hline B VS. A & Asian & 18 & $1.644^{*} 10^{-72}$ & 0.188 & 0.940 & $0.904(0.777-1.051)$ & $0.932(0.905-0.960)$ \\
\hline B VS. A & GC & 16 & $2.061 * 10^{-72}$ & 0.121 & 0.605 & $0.873(0.736-1.037)$ & $0.917(0.889-0.947)$ \\
\hline B VS. A & ESCC & 2 & 0.749 & 0.884 & 1.000 & $1.006(0.927-1.092)$ & $1.006(0.927-1.092)$ \\
\hline B VS. A & PB & 16 & $6.359^{*} 10^{-73}$ & 0.299 & 1.000 & $0.920(0.786-1.077)$ & $0.933(0.906-0.961)$ \\
\hline B VS. A & $\mathrm{HB}$ & 3 & 0.167 & 0.061 & 0.305 & $0.773(0.555-1.076)$ & $0.793(0.622-1.010)$ \\
\hline BB vs. AA & overall & 19 & $6.471 * 10^{-71}$ & 0.166 & 0.830 & $0.810(0.601-1.092)$ & $0.868(0.819-0.921)$ \\
\hline BB vs. AA & Asian & 18 & $1.516^{*} 10^{-71}$ & 0.191 & 0.955 & $0.816(0.602-1.106)$ & $0.869(0.819-0.922)$ \\
\hline BB vs. AA & GC & 16 & $2.211^{*} 10^{-71}$ & 0.127 & 0.635 & $0.763(0.539-1.080)$ & $1.013(0.859-1.196)$ \\
\hline BB vs. AA & ESCC & 2 & 0.765 & 0.875 & 1.000 & $0.844(0.792-0.899)$ & $1.013(0.859-1.196)$ \\
\hline BB vs. AA & PB & 16 & $4.978^{*} 10^{-72}$ & 0.300 & 1.000 & $0.845(0.615-1.161)$ & $0.872(0.822-0.925)$ \\
\hline BB vs. AA & $\mathrm{HB}$ & 3 & 0.182 & 0.088 & 0.440 & $0.592(0.286-1.224)$ & $0.630(0.371-1.071)$ \\
\hline BA vs. AA & overall & 19 & $2.232 * 10^{-20}$ & 0.192 & 0.960 & $0.900(0.768-1.054)$ & $0.946(0.899-0.996)$ \\
\hline BA vs. AA & Asian & 18 & $8.982^{*} 10^{-21}$ & 0.224 & 1.000 & $0.904(0.768-1.064)$ & $0.948(0.900-0.998)$ \\
\hline BA vs. AA & GC & 16 & $1.397^{*} 10^{-21}$ & 0.184 & 0.920 & $0.883(0.734-1.061)$ & $0.937(0.886-0.990)$ \\
\hline BA vs. AA & ESCC & 2 & 0.727 & 0.912 & 1.000 & $1.008(0.871-1.167)$ & $1.008(0.871-1.167)$ \\
\hline BA vs. AA & PB & 16 & $9.406^{*} 10^{-21}$ & 0.320 & 1.000 & $0.919(0.778-1.086)$ & $0.950(0.902-1.001)$ \\
\hline BA vs. AA & $\mathrm{HB}$ & 3 & 0.121 & 0.155 & 0.775 & $0.730(0.417-1.277)$ & $0.768(0.534-1.105)$ \\
\hline $\mathrm{BB}+\mathrm{BA}$ vs. $\mathrm{AA}$ & overall & 19 & $1.835^{*} 10^{-44}$ & 0.168 & 0.840 & $0.869(0.711-1.061)$ & $0.916(0.873-0.962)$ \\
\hline $\mathrm{BB}+\mathrm{BA}$ vs. $\mathrm{AA}$ & Asian & 18 & $5.451^{*} 10^{-45}$ & 0.195 & 0.975 & $0.873(0.71-1.072)$ & $0.918(0.874-0.963)$ \\
\hline$B B+B A$ vs. $A A$ & GC & 16 & $1.594^{*} 10^{-45}$ & 0.145 & 0.725 & $0.841(0.666-1.062)$ & $0.901(0.856-0.949)$ \\
\hline$B B+B A$ vs. $A A$ & ESCC & 2 & 0.707 & 0.892 & 1.000 & $1.010(0.879-1.159)$ & $1.010(0.879-1.159)$ \\
\hline $\mathrm{BB}+\mathrm{BA}$ vs. $\mathrm{AA}$ & PB & 16 & $5.135 * 10^{-45}$ & 0.309 & 1.000 & $0.895(0.723-1.108)$ & $0.920(0.876-0.966)$ \\
\hline$B B+B A$ vs. $A A$ & $\mathrm{HB}$ & 3 & 0.085 & 0.208 & 1.000 & $0.692(0.389-1.228)$ & $0.737(0.522-1.041)$ \\
\hline$B B$ vs. BA+AA & overall & 19 & $2.761^{*} 10^{-46}$ & 0.193 & 0.965 & $0.874(0.714-1.071)$ & $0.902(0.860-0.946)$ \\
\hline$B B$ vs. BA+AA & Asian & 18 & $7.547^{*} 10^{-47}$ & 0.217 & 1.000 & $0.878(0.715-1.079)$ & $0.902(0.860-0.947)$ \\
\hline BB vs. BA+AA & GC & 16 & $1.034^{*} 10^{-46}$ & 0.135 & 0.675 & $0.836(0.660-1.058)$ & $0.882(0.838-0.929)$ \\
\hline$B B$ vs. $B A+A A$ & ESCC & 2 & 0.892 & 0.917 & 1.000 & $1.007(0.883-1.148)$ & $1.007(0.883-1.148)$ \\
\hline BB vs. BA+AA & PB & 16 & $7.502^{*} 10^{-48}$ & 0.293 & 1.000 & $0.891(0.719-1.105)$ & $0.904(0.861-0.948)$ \\
\hline$B B$ vs. $B A+A A$ & $H B$ & 3 & 0.681 & 0.219 & 1.000 & $0.747(0.470-1.188)$ & $0.746(0.470-1.184)$ \\
\hline
\end{tabular}

$P_{H} P$ value of $\mathrm{Q}$ test for heterogeneity test, $P_{Z}$ means statistically significant, $P_{\text {Adjust }}$ Multiple testing $P$ value according to Bonferroni Correction, $H-B$ Hospital based, $P$ - $B$ Population based, HWE Hardy Weinberg Equilibrium; Note: Heterogeneity was considered to be significant when the $P$-value was less than 0.1. If there was no significant heterogeneity, a fixed effect model (Der-Simonian Laird) was used to evaluate the point estimates and $95 \% \mathrm{Cl}$; otherwise, a random effects model (Der-Simonian Laird) was used. And the Pz was calculated based on the actual model adopted. "*" indicated that $P_{\text {Adjust }}$ value less than 0.05 , and is considered as statistically significant 

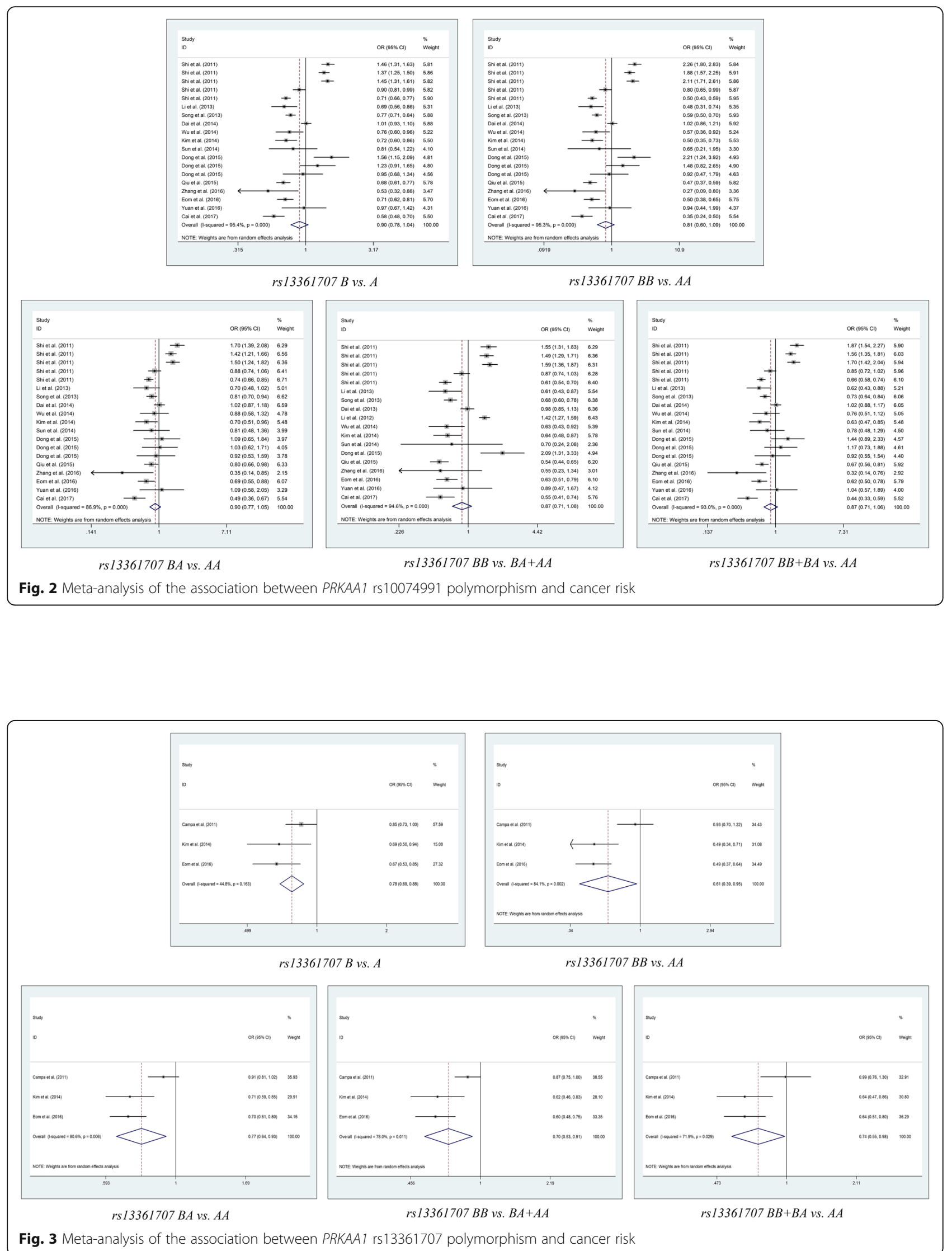

Fig. 3 Meta-analysis of the association between PRKAA1 rs13361707 polymorphism and cancer risk 


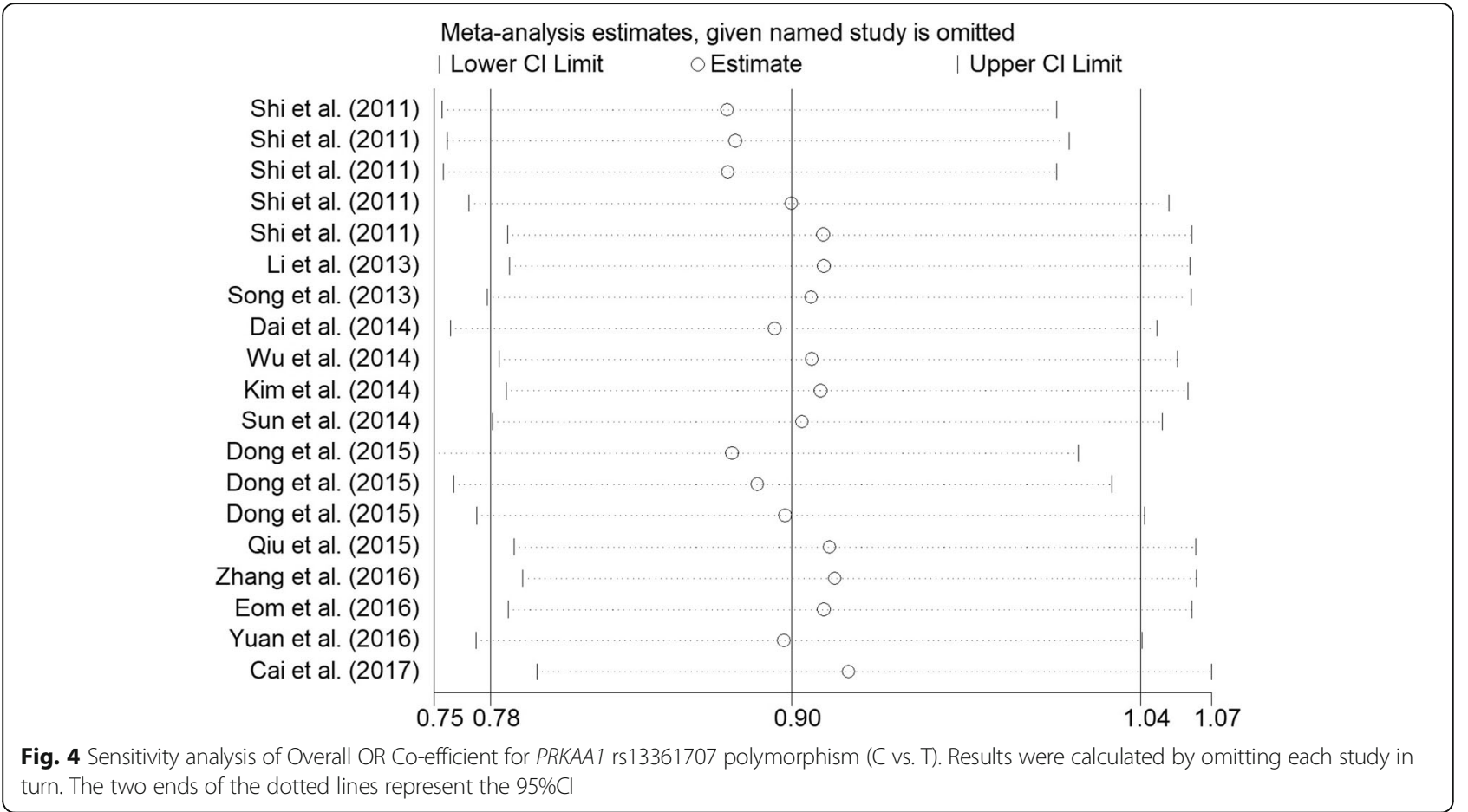

expression of PRKAA1 affects the overall survival (OS) and disease free survival (RFS) of these four cancers. The Kaplan-Meier estimate showed that there is no significant difference of OS and RFS between the low and high PRKAA1 TPM groups in gastric cancer (OS: Log-rank $P=0.48$, RFS: Log-rank $P=0.38$ ), breast cancer (OS: Log-rank $P=0.34$, RFS: Log-rank $P=0.98$ ), esophageal carcinoma (OS: Log-rank $P=0.096$, RFS: Log-rank $P=0.7$ ), and lung adenocarcinoma (OS: Log-rank $P=0.41$, RFS: Log-rank $P=0.20$ ).

\section{Discussion}

AMPK is a highly conserved heterotrimeric Ser/Thr kinase, it could phosphorylates many pivotal downstream targets through the increase ratio of AMP/ATP in environment, affect metabolic pathways of cell growth, cell cycle and autophagy [33, 34]. In normal cells, the ratio of AMP/ATP is in balance condition, when it is disrupted, many diseases will be followed, as well as malignant disease [35-37]. The underlying genetic mechanisms are still not clearly that how PRKAA1 gene affect the occurrence and development of cancer, one of them may be the activated AMPK and phosphorylated p53 induced cell cycle G1/S arrest, as well as influenced the cell cycle checkpoint [8]. On the other way, some publications reported that activated AMPK take pate in the activation of anti-inflammatory agents [38], as well as inhibition the inflammatory function of macrophage [39].The lack of anti-inflammatory function will occur with the allele mutation of PRKAA1 allele, and the patients would suffer from several epithelium disease [40]. What's more, another potential source of tumorigenesis is the bone marrow-derived cells, because they were reported could be recruited after epithelial damage [41]. Currently, several studies have demonstrated that PRKAA1 polymorphisms conduce to the development of gastric cancer, Sun et al. [42] revealed that LINC00152/ miR-139-5p could promote the cell glycolysis of gastric cancer cells through regulating PRKAA1 expression, $\mathrm{Li}$ et al. [43] indicated that Calcium Binding Protein 39-Like (CAB39L) may be a novel potential regulator for gastric cancer metabolism, which function on activation of PRKAA1/2.

There are several studies concerned about the polymorphisms of PRKAA1, and the variant sites include rs154268, rs3805486, rs461404, rs6882903, rs13361707 and rs10074991. After our comprehensive search and analyses, only rs13361707 and rs10074991 have 3 or more studies, so the other studies are excluded. Among these publications, the result is not consistent. $\mathrm{Li}$ et al. [9] and Eom et al. [10] suggested that rs13361707 polymorphism is remarkable related to an upgraded risk to gastric cancer in Asian and Caucasian, respectively. However, Sun et al. [13] and Yuan et al. [15] indicated the controversial result, in their study, rs13361707 of PRKAA1 doesn't affect the process of gastric cancer. On the meanwhile, the first three study of Shi et al. indicated that $\mathrm{T}$ allele of rs13361707 shows a significantly increasing risk of GC, however, Li et al., Kim et al. and Cai et al. revealed that the mutant allele of 


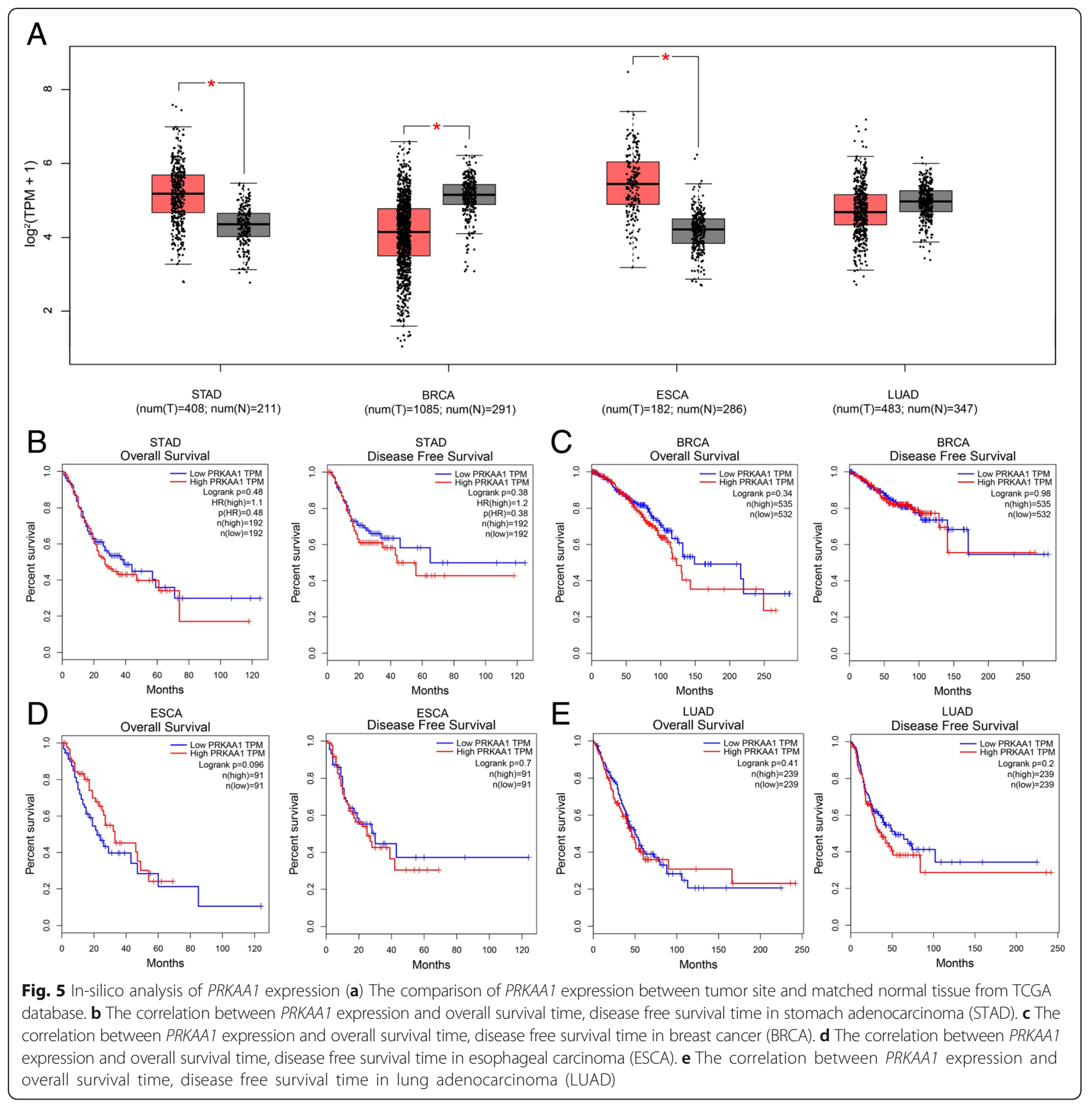

rs13361707 (C allele) caused enhanced risk of GC. In another case-control study conducted by Dai et al. [16], they revealed that the polymorphism of rs13361707 also doesn't work in esophageal squamous cell carcinoma. In current study, we identified that the rs13361707 polymorphism would not affect the susceptibility of cancer in the overall population. For rs10074991, Eom et al. [10] and Kim et al. [27] only reported partial increase cancer risks in several genetic models, while Campa et al. [12] revealed that rs10074991 is not associated with the tumorigenesis of breast cancer.
We revealed a significant decrease risk in allelic comparison model, heterozygote comparison model and dominant genetic model. Rs10074991 and rs13361707 polymorphisms both located at the intron of PRKAA1, within the perfect $L D\left(R^{2}=1.00\right)$. In a book named "Personalized Management of Gastric Cancer", Zhu et al. [44] demonstrated that the rs13361707 LD block mainly spans PTGER4, TTC33, and PRKAA1 gene, and a remarkable relationships between rs13361707 and these three genes were shown in the results from GTEx, so the polymorphism of rs13361707 might influence the expression of PRKAA1. In the current study, we enrolled 
all eligible case-control studies, from different race to different cancer types, aim to draw a systemic result for readers, and stratified analysis was also performed to avoid the bias. For example, the allele frequency of rs10074991, rs13361707 for all the enrolled Asian population based studies are ranged from 40 to $60 \%$, while the Caucasian population based studies are ranged about 80\% (Additional file 1: Table S1), it might cause by the ethnic difference. We exceed the Caucasian based study, and the overall result was not influenced (Table 2), but the future studies show focused on this difference between Asian and Caucasian. In the analysis of rs13361707, we also conducted the stratified analysis by the source of control, in order to reduce the heterogeneity of Q-test, however, the result in HB based subgroup or PB based subgroup is consistent with the result of overall pooled result. To confirm the result, large well-designed epidemiological studies based on population controls should be managed in the future.

There are several advantages of this meta-analysis. Initially, we enrolled all eligible studies focused on the relationships between PRKAA1 polymorphisms and overall cancer risks to conducted a comprehensive meta-analysis. Furthermore, NOS method was used to assess the quality of each accepted case-control study, and exceed the low quality studies to make sure the reliability of pooled result. Additionally, eligible studies were stratified and calculated in different subgroups to reduce the impact of heterogeneity, including subgroup of ethnicity, cancer type and source of control. Another point, the $P$ value of $\mathrm{Z}$ was adjusted by Bonferroni corrections $\left(P_{\text {Adjust }}\right)$, aim to avoiding the false positive results. Finally, the stability of results was assessed by sensitivity analysis, and potential publication bias was eliminating from the results of Egger's test and Begg's funnel plot.

However, there are also several disadvantages. To begin with, the untrustworthy result may be obtained, because of lack of subjects in several genetic polymorphisms. What's more, potential enrolled bias may be existed, due to that only publications written in English or Chinese were assessed. Then, most of the enrolled studies are concerned about GC, only 2 about ESCC, 1 about breast cancer, and 1 about lung cancer, therefore, the meta-analysis result might not be able to illustrated the impact of rs10074991 and rs13361707 in overall cancer risk. Last but not least, the striking level of heterogeneity between the enrolled publications might influence the result, although we conducted the meta-analysis with Der Simonian and Laird method.

\section{Conclusion}

Our data have successfully elaborated that PRKAA1 rs13361707 polymorphism is not participant with increased risk of cancer, while the A allele of PRKAA1 rs10074991 revealed a significant decrease risk, especially in Asian population. In the future., larger sample size studies based on numerous cancer types should be conducted to confirm the exploration of this issue.

\section{Additional file}

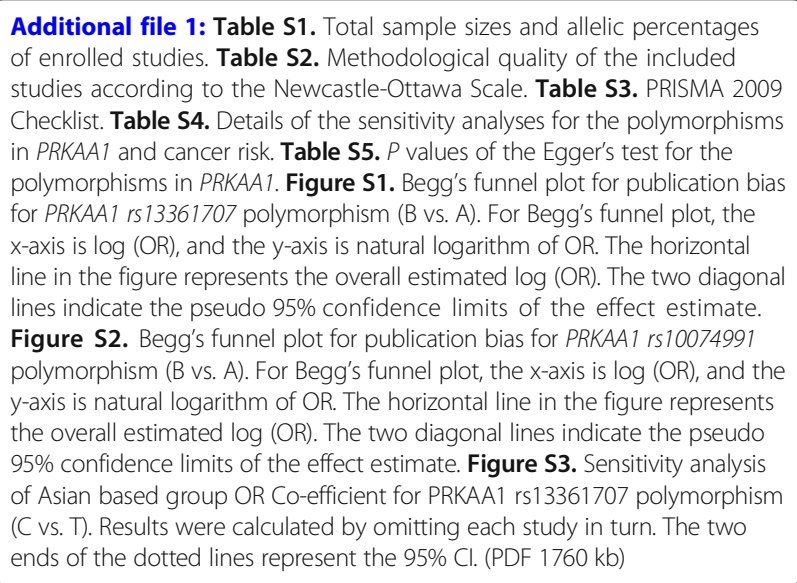

\section{Abbreviations}

AMP: Adenosine monophosphate; AMPK: AMP-activated protein kinase; ATP: Adenosine triphosphate; Cls: Confidence intervals; HWE: Hardy-Weinberg Equilibrium; mTORC1: Mammalian target of rapamycin complex 1; ORs: Odds ratio; SNP: Single nucleotide polymorphism; TPM: Transcripts Per Kilobase Million

\section{Funding}

This manuscript was supported by National Natural Science Foundation of China (81630019 to C Liang).

Availability of data and materials

All the data generated or analyzed in this study is included in this manuscript.

\section{Authors' contributions}

MJL and FXY accessed information from literature for this article. MJL, ZM and $\mathrm{HZY}$ designed and performed the meta-analysis. MJL and LCZ contributed towards writing, discussing, and editing the manuscript. All authors discussed the results and contributed to the final manuscript. All authors read and approved the final manuscript.

Ethics approval and consent to participate

Not applicable.

Consent for publication

Not applicable.

\section{Competing interests}

The authors declare that they have no competing interests.

\section{Publisher's Note}

Springer Nature remains neutral with regard to jurisdictional claims in published maps and institutional affiliations.

\section{Author details}

${ }^{1}$ Department of Urology, The First Affiliated Hospital of Anhui Medical University, No. 218th, Jixi Road, Hefei 230022, Anhui, China. ${ }^{2}$ Institute of Urology, Anhui Medical University, No. 218th, Jixi Road, Hefei 230022, Anhui, China. ${ }^{3}$ Anhui Province Key Laboratory of Genitourinary Diseases, Anhui Medical University, No. 218th, Jixi Road, Hefei 230022, Anhui, China. 
${ }^{4}$ Graduate School of Anhui Medical University, No. 81th, Meishan Road, Hefei 230032, Anhui, China.

\section{Received: 22 June 2018 Accepted: 4 October 2018} Published online: 19 October 2018

\section{References}

1. Arzumanyan A, Reis HM, Feitelson MA. Pathogenic mechanisms in HBV- and HCV-associated hepatocellular carcinoma. Nat Rev Cancer. 2013;13(2):123-35.

2. Cocoş R, Schipor S, Nicolae I, Thomescu C, Raicu F. Role of COX-2 activity and CRP levels in patients with non-melanoma skin cancer. -765G>C PTGS2 polymorphism and NMSC risk. Arch Dermatol Res. 2012;304(5):335.

3. Stapleton D, Mitchelhill Kl, Gao G, Widmer J, Michell BJ, Teh T, House CM, Fernandez CS, Cox T, Witters LA, et al. Mammalian AMP-activated protein kinase subfamily. J Biol Chem. 1996;271(2):611-4.

4. Towler MC, Hardie DG. AMP-activated protein kinase in metabolic control and insulin signaling. Circ Res. 2007;100(3):328

5. Hardie DG, Schaffer BE, Brunet A. AMPK: an energy-sensing pathway with multiple inputs and outputs. Trends Cell Biol. 2016;26(3):190-201.

6. Kahn BB, Alquier T, Carling D, Hardie DG. AMP-activated protein kinase: ancient energy gauge provides clues to modern understanding of metabolism. Cell Metab. 2005;1(1):15-25.

7. He XY, Liu XJ, Chen X, Bian LG, Zhao WG, Shen JK, Sun QF. Gambogic acid induces EGFR degradation and Akt/mTORC1 inhibition through AMPK dependent-LRIG1 upregulation in cultured U87 glioma cells. Biochem Biophys Res Commun. 2013;435(3):397-402.

8. Jones RG, Plas DR, Kubek S, Buzzai M, Mu J, Xu Y, Birnbaum MJ, Thompson CB. AMP-activated protein kinase induces a p53-dependent metabolic checkpoint. Mol Cell. 2005;18(3):283-93.

9. Li M, Huang L, Qiu H, Fu Q, Li W, Yu Q, Sun L, Zhang L, Hu G, Hu J. Helicobacter pylori infection synergizes with three inflammation-related genetic variants in the GWASs to increase risk of gastric cancer in a Chinese population. PLoS One. 2013;8(9):e74976.

10. Eom SY, Hong SM, Yim DH, Kwon HJ, Kim DH, Yun HY, Song YJ, Youn SJ, Hyun T, Park JS. Additive interactions betweenPRKAA1polymorphisms andHelicobacter pyloriCagA infection associated with gastric cancer risk in Koreans. Cancer Med. 2016;5(11):3236-335

11. Cai M, Dai S, Chen W, Xia C, Lu L, Dai S, Qi J, Wang M, Wang M, Zhou L. Environmental factors, seven GWAS-identified susceptibility loci, and risk of gastric cancer and its precursors in a Chinese population. Cancer Med. 2017; 6(3):708-20.

12. Campa D, Claus R, Dostal L, Stein A, Chang-Claude J, Meidtner K, Boeing H, Olsen A, Tjonneland A, Overvad K, et al. Variation in genes coding for AMPactivated protein kinase (AMPK) and breast cancer risk in the European prospective investigation on Cancer (EPIC). Breast Cancer Res Treat. 2011; 127(3):761-7.

13. Sun Y, Gu J, Ajani JA, Chang DW, Wu X, Stroehlein JR. Genetic and intermediate phenotypic susceptibility markers of gastric cancer in Hispanic Americans: a casecontrol study. Cancer. 2014;120(19):3040.

14. Wu D, Li Y. Association of Helicobacter pylori infection and polymorphisms pf PSCA, PLCE1 and PRKAA1 with gastric cancer susceptibility[D]. Lanzhou: Lanzhou University; 2014

15. JJ Y, YY L, TT T NL, Zhu Y, Zou J, Gao J, Shen L. Risk prediction for earlyonset gastric carcinoma: a case-control study of polygenic gastric cancer in Han Chinese with hereditary background. Oncotarget. 2016;7(23):33608-15.

16. Dai N, Zheng M, Wang C, Ji Y, Du J, Zhu C, He Y, Zhu M, Zhu X, Sun M. Genetic variants at $8 \mathrm{q} 24$ are associated with risk of esophageal squamous cell carcinoma in a Chinese population. Cancer Sci. 2014;105(6):731-5.

17. Bonferroni CE: Teoria Statistica Delle Classi e Calcolo Delle Probabilità. Comm Firenze. 1936;8(1936):3-62.

18. Handoll HH. Systematic reviews on rehabilitation interventions. Arch Phys Med Rehabil. 2006;87(6):875.

19. Dersimonian R, Laird N. Meta-analysis in clinical trials. Control Clin Trials. 1986;7(3):177.

20. Mantel N. Statistical aspects of the analysis of data from retrospective studies of disease. J Natl Cancer Inst. 1959;22(4):719-48.

21. Tobias A, Campbell M: Modeling influenza epidemics in the relation between black smoke and total mortality. 1999.

22. Egger M, Davey Smith G, Schneider M, Minder C. Bias in meta-analysis detected by a simple, graphical test. BMJ. 1997;315(7109):629-34.
23. Begg CB, Mazumdar M. Operating characteristics of a rank correlation test for publication bias. Biometrics. 1994;50(4):1088-101.

24. Tang Z, Li C, Kang B, Gao G, Li C, Zhang Z. GEPIA: a web server for cancer and normal gene expression profiling and interactive analyses. Nucleic Acids Res. 2017;45(W1):W98-W102.

25. Shi Y, Hu Z, Wu C, Dai J, Li H, Dong J, Wang M, Miao X, Zhou Y, Lu F. A genome-wide association study identifies new susceptibility loci for noncardia gastric cancer at 3q13.31 and 5p13.1. Nat Genet. 2011;43(12):1215-8.

26. Song HR, Kim HN, Kweon SS, Choi JS, Shim HJ, Sang HC, Chung IJ, Park YK, Kim SH, Choi YD. Genetic variations in the PRKAA1 and ZBTB20 genes and gastric cancer susceptibility in a Korean population. Mol Carcinog. 2013; 52(S1):155-60

27. Kim YD, Yim DH, Eom SY, Moon SI, Yun HY, Song YJ, Youn SJ, Hyun T, Park JS, Kim BS, et al. Risk of gastric cancer is associated with PRKAA1 gene polymorphisms in Koreans. World J Gastroenterol. 2014;20(26):8592-8.

28. Dong Y, Chen J, Chen Z, Tian C, Lu H, Ruan J, Yang W. Evaluating the Association of Eight Polymorphisms with Cancer susceptibility in a Han Chinese population. PLoS One. 2015;10(7):e0132797.

29. Zhang Y, Zhang C, Yu X, Xu X, Wang L, Geng C, Dong Q. The correlation between polymorphisms of PRKAA1 and UNC5CL and susceptibility to gastric cancer. J Chin Clin Doct. 2016;44(10):21-5.

30. Qiu LX, He J, Cheng L, Zhou F, Wang MY, Sun MH, Zhou XY, Li J, Guo WJ, Wang YN, et al. Genetic variant of PRKAA1 and gastric cancer risk in an eastern Chinese population. Oncotarget. 2015;6(40):42661-6.

31. Wells M, Chande N, Adams P, Beaton M, Levstik M, Boyce E, Mrkobrada M. Meta-analysis: vasoactive medications for the management of acute variceal bleeds. Aliment Pharmacol Ther. 2012;35(11):1267-78.

32. Moher D, Liberati A, Tetzlaff J, Altman DG, Group P. Preferred reporting items for systematic reviews and meta-analyses: the PRISMA statement. Int J Surg. 2010;8(5):336-41.

33. Carling D, Thornton C, Woods A, Sanders MJ. AMP-activated protein kinase: new regulation, new roles? Biochem J. 2012;445(1):11-27.

34. Igata M, Motoshima H, Tsuruzoe K, Kojima K, Matsumura T, Kondo T, Taguchi T, Nakamaru K, Yano M, Kukidome D. Adenosine monophosphate-activated protein kinase suppresses vascular smooth muscle cell proliferation through the inhibition of cell cycle progression. Circ Res. 2005;97(8):837.

35. Kim E, Park J, Lim S, Choi JW, Kim HS, Seok H, Seo JK, Oh K, Lee D, Kim KT. Activation of AMP-activated protein kinase is essential for lysophosphatidic acid-induced cell migration in ovarian Cancer cells. J Biol Chem. 2011; 286(27):24036

36. Krishan S, Richardson DR, Sahni S. Gene of the month. AMP kinase (PRKAA1). J Clin Pathol. 2014:67(9):758-63.

37. Ma X, Zhang J, Deng R, Ding S, Gu N, Guo X. Synergistic effect of smoking with genetic variants in the AMPKa1 gene on the risk of coronary artery disease in type 2 diabetes. Diabetes Metab Res Rev. 2015;30(6):483-8.

38. O'Neill LAJ, Hardie DG. Metabolism of inflammation limited by AMPK and pseudo-starvation. Nature. 2013;493(7432):346-55.

39. Sag D, Carling D, Stout RD, Suttles J. Adenosine 5'-monophosphate-activated protein kinase promotes macrophage polarization to an anti-inflammatory functional phenotype. J Immunol. 2008;181(12):8633.

40. Macdonald TT, Horton MA, Choy MY, Richman PI. Increased expression of laminin/collagen receptor (VLA-1) on epithelium of inflamed human intestine. J Clin Pathol. 1990:43(4):313-5.

41. Houghton J, Stoicov C, Nomura S, Rogers AB, Carlson J, Li H, Cai X, Fox JG, Goldenring JR, Wang TC. Gastric Cancer originating from bone marrowderived cells. Science (New York, NY). 2004;306(5701):1568.

42. Sun K, Hu P, Xu F. LINC00152/miR-139-5p regulates gastric cancer cell aerobic glycolysis by targeting PRKAA1. Biomed Pharmacother. 2018;97: 1296-302.

43. Li W, Wong CC, Zhang X, Kang W, Nakatsu G, Zhao Q, Chen H, Go MYY, Chiu PWY, Wang $X$, et al. CAB39L elicited an anti-Warburg effect via a LKB1AMPK-PGC1alpha axis to inhibit gastric tumorigenesis. Oncogene. 2018. https://doi.org/10.1038/s41388-018-0402-1.

44. Zhu M, Jin G. Genetics and Molecular Signature of Gastric Cancer. In: Wei J, Liu B, editors. Personalized Management of Gastric Cancer. Singapore: Springer; 2017. p. 15-33. 